\title{
Association of Skin Color, Race/Ethnicity, and Hearing Loss Among Adults in the USA
}

\author{
Frank R. Lin $^{1,2,7}$, Paige Maas ${ }^{3}$, Wade Chien $^{1}$, John P. Carey ${ }^{1}$, Luigi Ferrucci ${ }^{4}$, and Roland \\ THORPE 5,6 \\ ${ }^{1}$ Department of Otolaryngology-Head and Neck Surgery, Johns Hopkins School of Medicine, Baltimore, MD 21287, USA \\ ${ }^{2}$ Department of Epidemiology and Center on Aging and Health, Johns Hopkins Bloomberg School of Public Health, Baltimore, \\ MD 21205, USA \\ ${ }^{3}$ Department of Biostatistics, Johns Hopkins Bloomberg School of Public Health, Baltimore, MD 21205, USA \\ ${ }^{4}$ Longitudinal Studies Section, National Institute on Aging, Baltimore, MD 21225, USA \\ ${ }^{5}$ Johns Hopkins Center for Health Disparities Solutions, Johns Hopkins Bloomberg School of Public Health, Baltimore, MD 21205, USA \\ ${ }^{6}$ Department of Health Policy and Management, Johns Hopkins Bloomberg School of Public Health, Baltimore, MD 21205, USA \\ ${ }^{7}$ Department of Epidemiology and Center on Aging and Health, Johns Hopkins Bloomberg School of Public Health, 2024 \\ Monument St, Suite 2-700, Baltimore, MD 21287, USA
}

Received: 8 August 2011; Accepted: 19 October 2011; Online publication: 29 November 2011

\begin{abstract}
Epidemiologic studies of hearing loss in adults have demonstrated that the odds of hearing loss are substantially lower in black than in white individuals. The basis of this association is unknown. We hypothesized that skin pigmentation as a marker of melanocytic functioning mediates this observed association and that skin pigmentation is associated with hearing loss independent of race/ethnicity. We analyzed cross-sectional data from 1,258 adults (20-59 years) in the 2003-2004 cycle of the National Health and Nutritional Examination Survey who had assessment of Fitzpatrick skin type and pure-tone audiometric testing. Audiometric thresholds in the worse hearing ear were used to calculate speech- $(0.5-4 \mathrm{kHz})$ and high-frequency (3-8 kHz) pure-tone averages (PTA). Regression models were stratified by Fitzpatrick skin type or race/ethnicity to examine the association of each factor with hearing loss independent of the other. Models were adjusted for potential confounders (demographic, medical, and noise exposure covariates). Among all
\end{abstract}

Correspondence to: Frank R. Lin · Department of Epidemiology and Center on Aging and Health · Johns Hopkins Bloomberg School of Public Health - 2024 Monument St, Suite 2-700, Baltimore, MD 21287, USA. Telephone: +1-443-2876509; fax: +1-410-6149444; email: flin1@jhmi.edu participants, race/ethnicity was associated with hearing thresholds (black participants with the best hearing followed by Hispanics and then white individuals), but these associations were not significant in analyses stratified by skin color. In contrast, in race-stratified analyses, darker-skinned Hispanics had better hearing than lighterskinned Hispanics by an average of $-2.5 \mathrm{~dB}$ hearing level (HL; $95 \% \mathrm{CI},-4.8$ to -0.2$)$ and $-3.1 \mathrm{~dB}$ HL $(95 \% \mathrm{CI}$, -5.3 to -0.8 ) for speech and high-frequency PTA, respectively. Associations between skin color and hearing loss were not significant in white and black participants. Our results demonstrate that skin pigmentation is independently associated with hearing loss in Hispanics and suggest that skin pigmentation as a marker of melanocytic functioning may mediate the strong association observed between race/ethnicity and hearing loss.

Keywords: hearing loss, race, melanocytes, epidemiology

\section{INTRODUCTION}

Epidemiologic studies of large population-based cohorts have consistently demonstrated lower rates 
of hearing loss among black than white participants with the odds of hearing loss generally $40-60 \%$ lower in black individuals (Cooper 1994; Helzner et al. 2005; Lin et al. 2011b). These findings have also been corroborated in smaller epidemiological studies that have investigated rates of hearing loss in workers exposed to similar levels of occupational noise. In these studies of firefighters (Jerger et al. 1986) and metal fabricating workers (Ishii and Talbott 1998), black workers had lower rates of hearing loss compared with white workers. These latter findings among black and white adults of similar occupational and socioeconomic background suggest a possible biological basis for the association of race with hearing loss susceptibility.

A number of mechanisms may be theoretically implicated in the observed association between race and hearing loss. Melanocytes, which produce the melanin pigment that determines skin color (Taylor 2002), are present in both the skin and cochlea, and one temporal bone histology study has suggested that there may be concordance in the degree of skin and cochlear pigmentation (Wolff 1931). Increased melanin in the inner ear may subsequently protect the cochlea against age-related cellular declines and hearing loss in darkerskinned individuals (Hood et al. 1976; Attias and Pratt 1985; Ohlemiller et al. 2009a, b). Other factors that could be possibly associated with race such as differences in noise exposure or in genetic determinants of hearing loss could also explain the epidemiologic association of race and hearing loss (Lin et al. 2011b).

The objective of the current study was to determine if skin color is associated with hearing loss independent of race/ethnicity. We hypothesized that darker skin color as characterized by Fitzpatrick skin type is associated with better hearing (lower hearing thresholds) independent of race, and conversely that race/ethnicity is not associated with hearing loss after adjustment for skin color. Determining whether skin color and hence melanocytic functioning is independently associated with hearing loss may provide new insights into the pathophysiology and treatment of hearing loss.

\section{METHODS}

\section{Study cohort}

The National Health and Nutritional Examination Surveys (NHANES) are an ongoing program of studies designed to assess the health, functional, and nutritional status of the civilian, non-institutionalized US population. Each sequential cross-sectional study uses a complex sampling design to survey a sample of the population, with selective oversampling of lowincome individuals, racial minorities, and older adults. Sampling weights allow for analyses that account for the complex sampling survey and yield results that are generalizable to the US population (Centers for Disease Control and Prevention 2005).

We used data from the 2003-2004 cycle, the only cycle in which both audiometric and dermatologic testing were conducted concurrently as part of the medical examination component of the NHANES protocol. Audiometric examinations were administered to half of all adult NHANES study participants 20-69 years, and dermatologic examinations were administered to all participants $20-59$ years.

\section{Audiometric assessment}

Audiometry was performed by a trained examiner according to established NHANES protocols (Centers for Disease Control and Prevention 2004b). Briefly, air conduction hearing thresholds were obtained from both ears in a dedicated, sound-isolating room in the mobile examination center. Testing was conducted according to a modified Hughson Westlake procedure using the automated testing mode of the audiometer (Interacoustics AD226) and/or manually per the testing protocol. Quality assurance and quality control were established through daily calibration of equipment and monitoring of ambient noise levels using a sound level meter. The audiometric test room met or exceeded ANSI S3.1-1991 guidelines for maximum permissible ambient noise levels. Air conduction stimuli were presented primarily through supra-aural earphones (TDH 39P). Insert earphones (ER3A) were reserved for cases of collapsing ear canals or for a cross-over retesting protocol in cases of asymmetric hearing loss (masking was not performed). As an additional quality measure, thresholds were measured twice at $1 \mathrm{kHz}$ in both ears, and audiometry was repeated if there was $>10 \mathrm{~dB}$ discrepancy between the threshold measurements. Hearing thresholds from 0.5 to $8 \mathrm{kHz}$ in the worse hearing ear, using the first threshold tested at $1 \mathrm{kHz}$ and incorporating manual re-test thresholds as needed were used in all analyses. A speech-frequency PTA was defined as an average of hearing thresholds at $0.5,1,2$, and $4 \mathrm{kHz}$ and a high-frequency PTA was defined as an average of hearing thresholds at 3, 4, 6, and $8 \mathrm{kHz}$ in the worse hearing ear. All hearing thresholds in this manuscript are reported as $\mathrm{dB}$ hearing level (HL) (ANSI 2004).

\section{Dermatologic assessment}

The dermatologic exam in NHANES consisted of four images of each study participant taken using a highresolution digital camera (Nikon DCS 760) under standardized studio photographic conditions in a customized dermatology exam room of the NHANES mobile examination center (Centers for Disease 
Control and Prevention 2004a, 2004c). Photographed body sites included the participant's back, elbows, inner arm, front of legs and hands, and back of legs with palms. Image quality control measures were initiated before every session by capturing an image of a color card comprising 24 scientifically prepared colored squares. Images were digitized and delivered weekly to two dermatologists who reviewed all images for various dermatologic conditions (Fitzpatrick skin type, psoriasis, and dermatitis).

The Fitzpatrick classification is the most common method of classifying skin type (Fitzpatrick 1988; Roberts 2009). Two dermatologists (readers 1 and 2) blinded to demographic and racial data on study participants rated the standardized photographs according to the following established Fitzpatrick scale:

1. Always burns, never tans (pale white skin)

2. Always burns easily, tans minimally (white skin)

3. Burns moderately, tans uniformly (light brown skin)

4. Burns minimally, always tans well (moderate brown skin)

5. Rarely burns, tans profusely (dark brown skin)

6. Never burns (deeply pigmented dark brown to black skin)

A priori, we chose to use Fitzpatrick ratings from reader 2 in all analyses in order to maintain the consistency of the ordinal Fitzpatrick scale across all analyses. Agreement between readers 1 and 2 was moderate (absolute agreement, $61.9 \%$; kappa $=0.46$ ). Ratings from reader 1 were used in a final set of analyses in order to confirm the consistency of our findings.

\section{Other study variables}

Data on demographic variables, history of noise exposure, and medical history were obtained from interviews. Self-reported race/ethnicity was grouped as non-Hispanic white (white), non-Hispanic black (black), or Mexican-American/other Hispanic (Hispanic). Education and household income were collapsed into a fourand three-level variable, respectively (Table 1 ). Variables related to medical history included diabetes (based on self-reported diagnosis and/or current use of insulin or other diabetic medications), smoking (current/former/ never), hypertension (told by physician on two or more visits about hypertension diagnosis), and stroke (selfreported history). Noise exposure history incorporated assessment (yes/no) of firearm use ("Have you ever used firearms for target shooting, hunting, or other purposes?"), occupational noise ("Have you ever had exposure to loud noise for $\geq 5 \mathrm{~h}$ /week?"), and leisure noise ("Have you ever had exposure to steady loud noise or music for $\geq 5 \mathrm{~h}$ /week?”).

\section{Statistical analysis}

We accounted for the complex sampling design in all analyses by using sample weights according to National Center for Health Statistics guidelines except for Table 1 (Guidelines). The purpose of Table 1 was only to give descriptive statistics on the characteristics of the study cohort rather than nationally generalizable estimates and hence weights were excluded. The significance of proportional differences in factors across racial/ethnic groups was determined with a Chi-square test. Audiograms were plotted using unadjusted, weighted mean estimates of hearing thresholds at each pure-tone frequency. Linear regression analyses were used to investigate the association between hearing thresholds at each pure-tone frequency or PTA and the covariate(s) of interest while adjusting for potential confounders. The $\beta$-coefficients from these regressions are interpreted as the average difference in hearing threshold (in $\mathrm{dB} \mathrm{HL}$, positive values indicate greater hearing loss and negative values indicate less hearing loss) per unit difference in the studied covariate. Per NCHS guidelines, the Taylor Series Linearization method was used for variance estimation. All analyses were conducted using STATA 11.0 (StataCorp, College Station, TX) and $\mathrm{R}$ version 2.12.0 (R Foundation, Vienna, Austria), and two-sided $p$ values of $<.05$ were considered statistically significant.

\section{RESULTS}

\section{Study cohort}

Demographic, medical, and noise exposure characteristics differed across self-reported racial/ethnic groups with white participants generally having higher education and income, and greater noise exposure than black or Hispanic participants (Table 1). Fitzpatrick skin type also varied by racial/ethnic grouping with white individuals primarily classified as Fitzpatrick 2-3, black individuals as Fitzpatrick 5-6, and Hispanic individuals as Fitzpatrick 3-4.

\section{Hearing loss by race/ethnicity}

The mean audiograms for each racial/ethnic group are displayed in Figure 1. Hearing thresholds for pure tones from 3 to $8 \mathrm{kHz}$ differed significantly by race/ethnicity with black participants, on average, having the best hearing (lowest hearing thresholds) followed by Hispanics and then white individuals having the worse hearing (greatest hearing thresholds) after adjusting for all demographic (age, sex, education, and income), medical, and noise exposure covariates. 
TABLE 1

Characteristics by race/ethnicity of participants with both audiometric and Fitzpatrick skin type testing, NHANES 2003-2004, $n=1,258$

White $(\mathrm{N}=674) \quad$ Black $(\mathrm{N}=289) \quad$ Hispanic $(\mathrm{N}=295) \quad$

Demographics

Age (years)

$\begin{array}{ll}20-29 & 187(0.28) \\ 30-39 & 171(0.25) \\ 40-49 & 158(0.23) \\ 50-59 & 158(0.23) \\ \text { Female } & 358(0.53)\end{array}$

$92(0.32)$

$69(0.24)$

$80(0.28)$

$48(0.17)$

$155(0.54)$

$60(0.09)$

$172(0.25)$

$254(0.38)$

$188(0.28)$

Some college

College graduate or above

Income

$<\$ 25 \mathrm{~K}$

$\$ 25 \mathrm{~K}$ to $<\$ 55 \mathrm{~K}$

$139(0.21)$

$191(0.30)$

$\geq \$ 55 \mathrm{~K}$

$314(0.49)$

Medical history

Diabetes

$32(0.05)$

$18(0.06)$

.36

Smoking

Never

Former

Current

Hypertension

Stroke

$317(0.47)$

$140(0.21)$

$217(0.32)$

$115(0.17)$

$11(0.02)$
$20(0.07)$

$70(0.24)$

$73(0.25)$

$100(0.35)$

$46(0.16)$

$81(0.30)$

$96(0.35)$

$94(0.35)$

$168(0.58)$

$37(0.13)$

$84(0.29)$

$53(0.18)$

$5(0.02)$ $\begin{array}{rr}85 & (0.29) \\ 82 & (0.28) \\ 82 & (0.28) \\ 46 & (0.15) \\ 153 & (0.52)\end{array}$

.05

.90

$141(0.48)$

$65(0.22)$

$61(0.21)$

28 (0.09)

$99(0.36)$

$114(0.41)$

$65(0.23)$

$<.001$

Noise exposure

Work noise

Leisure noise

$261(0.39)$

$217(0.32)$

$62(0.09)$

$79(0.28)$

$77(0.27)$

$10(0.03)$

$96(0.35)$

$67(0.22)$

$10(0.03)$

.005

.007

$<.001$

Fitzpatrick skin type

1
2
3
4
5
6

$33(0.05)$

$263(0.39)$

$346(0.51)$

$31(0.05)$

$0(0)$

$1(0)$

All values are $n$ (proportion)

\section{Hearing loss by Fitzpatrick skin type}

Mean audiograms stratified by Fitzpatrick skin type demonstrated that individuals with darker skin types generally had better hearing than individuals with lighter skin types with significant associations between skin type and hearing thresholds observed at 4 and $6 \mathrm{kHz}$ (Fig. 2a). Collapsing Fitzpatrick skin type into a binary variable (Fitzpatrick types 1-3 versus 4-6) demonstrated stronger associations 


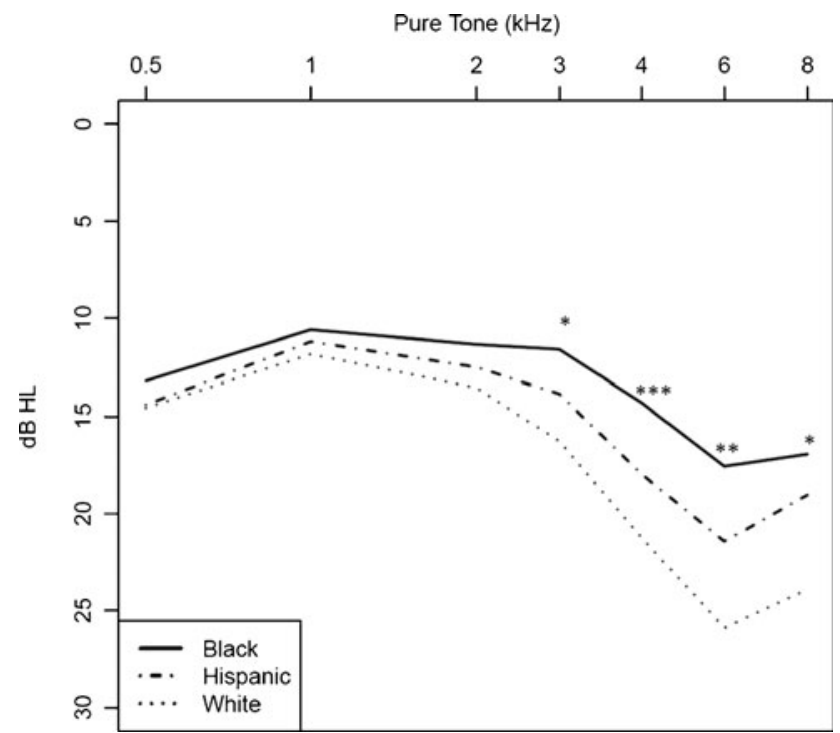

FIG. 1. Mean Audiograms by Race/Ethnicity. The significance of the association of racial/ethnic category with each pure tone is denoted by astericks $\left({ }^{*} P<.05\right.$; $\left.{ }^{* *} P<.01 ;{ }^{* * *} P<.001\right)$ based on regression models adjusting for all demographic (age, sex, education, income), medical (diabetes, smoking, hypertension, stroke), and noise (work, leisure, firearm) exposure covariates.

between Fitzpatrick skin type and hearing thresholds. Mean audiograms from those participants with darker skin (Fitzpatrick 4-6) had better hearing thresholds across all pure-tone frequencies after adjustment for demographic (age, sex, education, and income), medical, and noise exposure covariates (Fig. 2b).

A

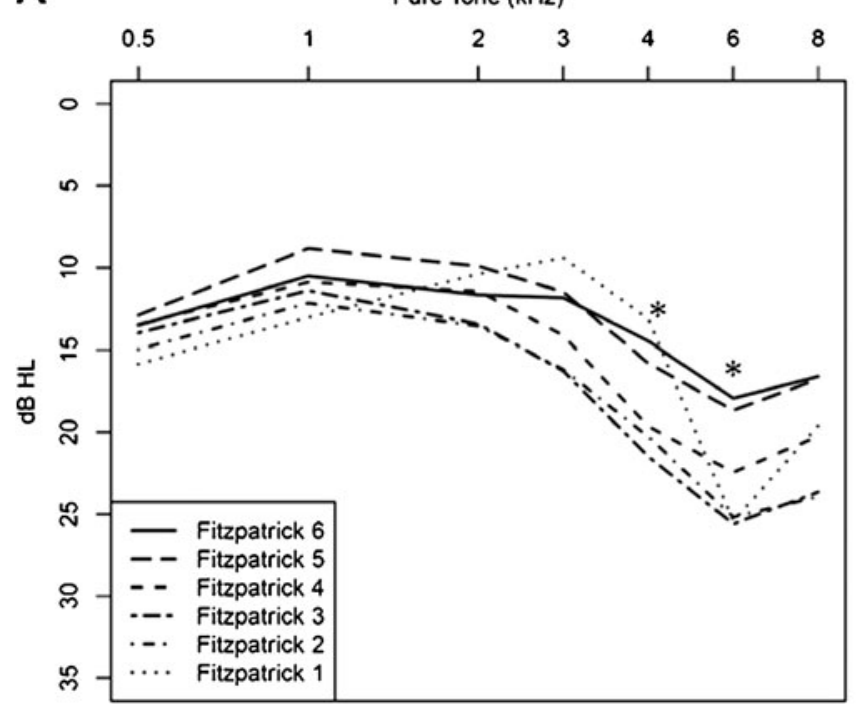

FIG. 2. Mean Audiograms by Fitzpatrick Skin Type (A) Mean audiograms by Fitzpatrick skin type 1-6. (B) Mean audiograms by binary Fitzpatrick skin type (type 1-3 vs. 4-6). The significance of the association of categorical Fitzpatrick skin type with each pure tone
Association of race/ethnicity with hearing loss independent of skin color

In order to characterize the association of race/ ethnicity with hearing loss independent of skin color, we stratified our analyses by Fitzpatrick skin type (Table 2). In contrast to the strong association between race/ethnicity and hearing loss in unstratified analyses (Fig. 1), there was no consistent association between race/ethnicity and speech- or high-frequency PTA after stratification by skin color (Table 2) and adjustment for demographic (age, sex, education, and income), medical, and noise exposure covariates.

\section{Association of skin color with hearing loss} independent of race/ethnicity

The association of Fitzpatrick skin type and hearing loss was examined in race-stratified analyses to determine the effects of skin color independent of race. Within each race/ethnicity, individuals were stratified into a lighter and darker group by using a Fitzpatrick cutpoint that would divide the subcohort into two approximately equal-sized groups. Hispanic individuals were stratified by Fitzpatrick 1-3 $(n=173)$ and Fitzpatrick $4-6 \quad(n=122)$, white individuals by Fitzpatrick 1-2 $(n=296)$ and Fitzpatrick 3-4 ( $n=377$; the one individual of white race assigned a Fitzpatrick skin type of 6 was excluded), and blacks by Fitzpatrick 2-5 $(n=75)$ versus Fitzpatrick $6(n=214)$.

For Hispanic-stratified analyses, the darker skin group had consistently better hearing than the

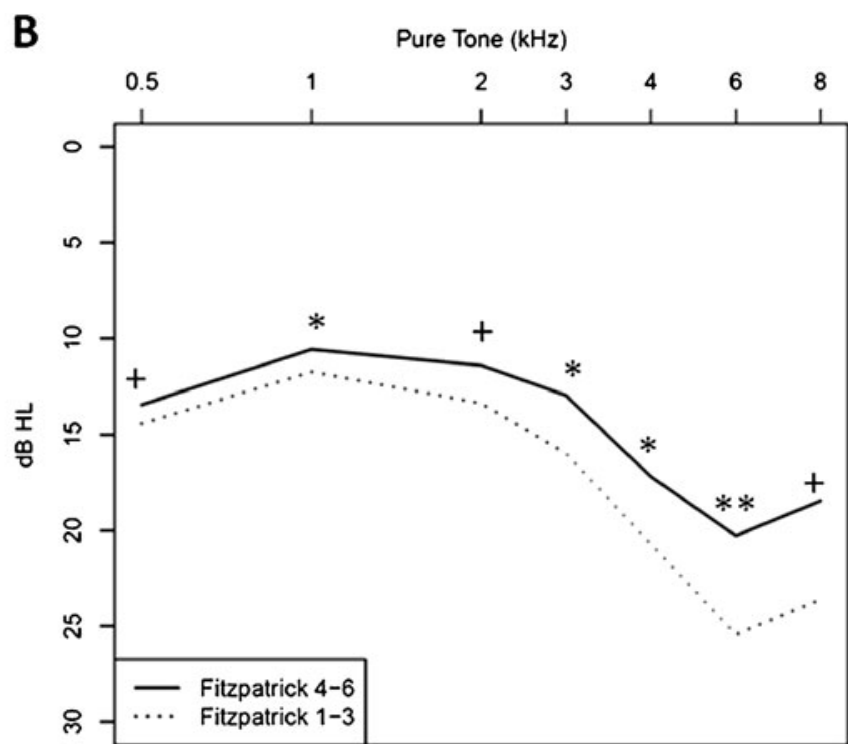

threshold is denoted by astericks $(+P<.10$; $P<.05$; ** $P<.01$; *** $P$ $<$.001) based on models adjusting for all demographic (age, sex, education, income), medical, and noise exposure covariates. 
TABLE 2

Association of speech-frequency and high-frequency pure-tone averages (PTA) with race/ethnicity after stratification by Fitzpatrick skin type

\begin{tabular}{|c|c|c|c|}
\hline \multicolumn{2}{|c|}{ Speech-frequency PTA $(0.5-4 \mathrm{kHz})$} & \multicolumn{2}{|c|}{ High-frequency PTA $(3-8 \mathrm{kHz})$} \\
\hline$\beta(95 \% C l) d B H L$ & $P$ & $\beta(95 \% \mathrm{Cl}) d B \mathrm{HL}$ & $P$ \\
\hline
\end{tabular}

Fitzpatrick skin type 2

White $(n=263)$

Hispanic $(n=35)$

Reference

$1.6(-3.6-6.9)$

52

Reference

$-0.4(-8.5-7.8)$

.98

Reference

0.1 (-3.8-4.0)
.03 (-2.5-2.5)

.96

Reference

$-1.6(-5.1-2.0)$

$-1.6(-4.4-1.1)$
Reference

-0.5 (-4.6-3.6)

$-0.7(-4.9-3.4)$
.79

.71

Hispanic $(n=108)$

$\beta$-Coefficients represent the mean difference in PTA between the specified racial/ethnic group and white participants (reference) after adjustment for demographic (age, sex, education, and income), medical, and noise exposure covariates

lighter-skinned group for both speech-frequency $(p=.04)$ and high-frequency PTA $(p=.01)$ after adjustment for demographic (age, sex, education, and income), medical, and noise exposure covariates. In this adjusted analysis, Hispanics with darker skin had on average hearing thresholds that were better by $-2.5 \mathrm{~dB}$ HL $(95 \% \mathrm{CI},-4.8$ to -0.2$)$ and $-3.1 \mathrm{~dB}$ HL $(95 \% \mathrm{CI},-5.3$ to -0.8$)$ for speech- and high-frequency PTA, respectively, than
Hispanics with lighter skin. We confirmed these results using Fitzpatrick skin ratings from reader 1, and the findings were qualitatively unchanged (c.f. Table 3) with the exception that these results only approached significance ( $p=.09$ and .07 for speechand high-frequency PTA, respectively). Associations between darker skin color and better hearing were not significant in white and black individuals (Table 3).

TABLE 3

Association of speech-frequency and high-frequency pure-tone averages (PTA) with skin color after stratification by race/ethnicity

\begin{tabular}{cccc}
\hline Speech-frequency PTA $(0.5-4 \mathrm{kHz})$ & High-frequency PTA (3-8 kHz) \\
\cline { 2 - 3 } & $\beta(95 \% \mathrm{Cl}) \mathrm{dB} \mathrm{HL}$ & $\beta(95 \% \mathrm{Cl}) \mathrm{dB} \mathrm{HL} \quad P$ \\
\hline
\end{tabular}

White $^{\mathrm{a}}$

Lighter skin $(n=296)$

Darker skin $(n=377)$

Black $^{\text {b }}$

Lighter skin $(n=75)$

Darker skin $(n=214)$

Reference

$0.8(-0.9-2.5)$
Reference

$-1.1(-4.1-1.8)$

Hispanic ${ }^{c}$

Lighter skin $(n=173)$

Darker skin $(n=122)$

Reference $-2.5(-4.8$ to -0.2$)$ .04

Reference $-3.1(-5.3$ to -0.8$)$ .01

$\beta$-Coefficients represent the mean difference in PTA between darker vs. lighter-skinned (reference group) individuals of the same racial/ethnic group after adjustment for demographic (age, sex, education, income), medical, and noise exposure covariates

aLighter skin, Fitzpatrick 1-2; darker skin, Fitzpatrick 3-4

bighter skin, Fitzpatrick 2-5; darker skin, Fitzpatrick 6

'Lighter skin, Fitzpatrick 1-3; darker skin, Fitzpatrick 4-6 
We performed additional analyses to test whether age moderates the association between skin color and hearing loss by stratifying analyses in Table 3 by age (20-39 years versus 40-59 years). In Hispanics, darker skin color was associated with better hearing in the older cohort with mean PTA differences greater than seen in the pooled age sample in Table 3. Darkerskinned older Hispanics (40-59 years) had on average hearing thresholds that were better by $-4.6 \mathrm{~dB}(95 \%$ CI, -8.5 to $-0.62, p=.03)$ and $-5.5 \mathrm{~dB}(95 \% \mathrm{CI},-11.6$ to $0.59, p=.07$ ) for speech-frequency and high-frequency PTA, respectively, than lighter-skinned older Hispanics. Skin color was not associated with hearing thresholds in younger Hispanics or in younger or older white and black individuals (data not shown).

\section{DISCUSSION}

\section{Summary of findings}

In this study using nationally representative NHANES data from 2003 to 2004, darker skin color as assessed by Fitzpatrick skin type was independently associated with better hearing thresholds in Hispanic individuals. In contrast, race/ethnicity was not associated with hearing thresholds after stratification by skin color. These results provide preliminary evidence that skin color is independently associated with hearing loss and that skin color as a marker of melanocytic functioning may mediate the strong association between race and hearing loss observed in previous epidemiological studies.

\section{Potential mechanistic pathways}

The findings from this study contribute to the literature examining the association between race and hearing loss. Previous reports from large epidemiological studies (Cooper 1994; Helzner et al. 2005; Agrawal et al. 2008; Lin et al. 2011a, b) and from smaller case-control studies of workers with occupational noise exposure (Jerger et al. 1986; Ishii and Talbott 1998) have consistently demonstrated better hearing thresholds in black compared with white participants. A number of explanatory factors may be theoretically implicated to explain the observed association between race and hearing loss in these studies. An earlier study conducted in 1931 has suggested that there may be concordant skin and cochlear pigmentation (Wolff 1931) with increased cochlear melanin pigmentation being associated with protection against hearing loss (Ohlemiller et al. 2009a). Alternatively, inter-racial/ethnic differences in autosomal or mitochondrial genetics not associated with skin color could also impart differential susceptibility to hearing loss. Finally, racial disparities in environmental factors such as noise exposure could also explain the observed association.

The findings of the current study suggest that skin color and hence melanocyte functioning may be the mechanism underlying the association between race and hearing loss. The melanin produced by strial melanocytes (intermediate cells) in the cochlea has been hypothesized to serve a protective role as a free radical scavenger, metal chelator, or regulator of calcium homeostasis in the stria vascularis, which is involved with generating and maintaining the endolymphatic potential necessary for normal hearing (Riley 1997; Murillo-Cuesta et al. 2010). A recent study has also demonstrated that deficiency in strial melanin is associated with marginal cell loss and decline in the endocochlear potential (Ohlemiller et al. 2009a). Melanin is also present throughout other parts of the cochlea including the Organ of Corti and modiolus (Wolff 1931) raising the possibility that melanin could exert effects beyond the stria vascularis. The role of melanocytes serving a protective role in the inner ear is supported by other studies that have demonstrated that individuals with lighter irises (as an indicator of cochlear pigmentation) (Bonaccorsi 1965; Hood et al. 1976; Attias and Pratt 1985; Barrenas and Hellstrom 1996) or with albinism or other pigmentary abnormalities are at increased risk for hearing loss (Creel et al. 1980; Garber et al. 1982; Toriello 2002; Hong et al. 2009).

Race-stratified analyses demonstrated that darker-skinned Hispanics had significantly better hearing than lighter-skinned Hispanics, but no consistent associations between skin color and hearing loss were observed in white or black individuals. There are several possible explanations for this lack of association in white and black racestratified analyses. First, there may be a non-linear threshold effect in the association of skin color with hearing loss such that only the darkest skin types (i.e., Fitzpatrick 4-6) are uniformly associated with better hearing. Consequently, an association between skin type and hearing loss would only be observed among Hispanics, among whom there are a sufficient number of individuals of both binary Fitzpatrick skin types. A second possibility is that skin color ratings by Fitzpatrick skin type are insufficiently precise to clearly distinguish between individuals of similar tonal skin color. As a result, in whites and blacks, among whom there is greater homogeneity in skin color compared with Hispanics (Table 1), the use of the Fitzpatrick scale may not precisely distinguish between individuals of different skin color. This misclassification would tend to bias any association of skin color with hearing loss toward the null hypothesis. Finally, the association of darker skin color with better hearing in Hispanics may 
itself be spurious and an example of a Type 1 error. However, the observed finding in Hispanics was consistent with our a priori hypothesis.

\section{Association of skin color with cochlear histology}

There has been little research examining the structural and functional characteristics of the cochlea in darker versus lighter-skinned individuals. Such studies are dependent on temporal bone histopathologic specimens, and most existing temporal bone collections do not contain information on race (personal communication with S. Merchant). One study published in 1931 demonstrated a concordance of black race with increased melanocytic pigmentation in the cochlea based on light microscopy (Wolff 1931), but results from this study are methodologically limited. To the authors' knowledge, no further studies have investigated the role of race and skin color with human cochlear histopathology. Animal studies have typically served as an adjunctive approach to studying this question, but the association of coat color, cochlear pigmentation, and hearing loss in mice and other animal models remains unclear (Conlee et al. 1986, 1988, 1989, 1994; Bartels et al. 2001; Ohlemiller et al. 2009a, b). However, many of these prior experiements have been confounded by comparing animals of different genetic backgrounds, and recent work using coisogenic mouse strains has demonstrated that the absence of strial melanin is strongly associated with marginal cell loss and endocochlear potential decline (Ohlemiller et al. 2009a). Further research analyzing human temporal bone specimens with electron microscopic and immunohistochemical techniques will be needed to definitively determine the structural differences in cochlear histopathology between darker and lighter-skinned individuals and to further refine these hypotheses.

\section{Limitations}

This study has limitations. The Fitzpatrick scale relies on a dermatologist's review of a series of digital photographs and may be imprecise, particularly when distinguishing between individuals of similar tonal skin color. However, a non-differential misclassification of participants' skin color would likely bias any observed results toward the null hypothesis, whereas we found strong associations between skin color and hearing loss in Hispanics. These results were also generally consistent regardless of whether skin type ratings from reader 1 or 2 were used. Further research using a quantitative method of skin color assessment that directly reflects skin melanin content (Gonzalez et al. 2010) will be needed to characterize the exact nature of the association between skin color and hearing loss. Residual confounding by other environmental or demographic variables or by inaccurate assessment of self-reported noise exposure is also a possibility. However, we have adjusted for those factors known to be associated with hearing loss, and the association with skin color remains robust.

\section{CONCLUSION}

The possibility of concordant skin and cochlear melanocyte functioning underlying the basis of black individuals having a substantially lower odds of hearing loss is intriguing. This finding may open the door to pharmacologically targeting cochlear melanocytes (intermediate cells) to possibly prevent or delay age-related hearing loss. Synthetic analogs of melanocyte stimulating hormone (MSH) have already been developed for human use (Abdel-Malek 2010), and animal studies have demonstrated a protective role of MSH against ototoxicity from cisplatin in which the mechanism remains unclear (Wolters et al. 2003, 2004). Current advances in administering drugs directly to the middle and inner ear via transtympanic injections (Bowe and Jacob 2010) also raise the possibility of precisely targeting pharmacological agents to the inner ear without systemic effects.

\section{REFERENCES}

Abdel-Malek ZA (2010) Development of alpha-melanocortin analogs for melanoma prevention and targeting. Adv Exp Med Biol 681:126-132

Agrawal Y, Platz EA, Niparko JK (2008) Prevalence of hearing loss and differences by demographic characteristics among US adults: data from the National Health and Nutrition Examination Survey, 1999-2004. ArchInternMed 168:1522-1530

Attias J, Pratt H (1985) Auditory-evoked potential correlates of susceptibility to noise-induced hearing loss. Audiology 24:149156

Barrenas ML, Hellstrom PA (1996) The effect of low level acoustic stimulation on susceptibility to noise in blue- and brown-eyed young human subjects. Ear Hear 17:63-68

Bartels S, Ito S, Trune DR, Nuttall AL (2001) Noise-induced hearing loss: the effect of melanin in the stria vascularis. HearRes 154:116-123

Bonaccorsi P (1965) The color of the iris as a "test" in the quantitative estimation, in man, of the melanin concentration in the stria vascularis. Ann Laringol Otol Rinol Faringol 64:725738

Bowe SN, JACOB A (2010) Round window perfusion dynamics: implications for intracochlear therapy. Curr Opin Otolaryngol Head Neck Surg 18:377-385

Centers for Disease Control and Prevention (2004a). National Centers for Health Statistics. The National Health and Nutrition Examination Survey. Dermatology examination data 2003-2004. 
Available at http://www.cdc.gov/nchs/data/nhanes/ nhanes_03_04/DEX_C.pdf. Accessed 3/1/2011

Centers for Disease Control and Prevention (2004b). National Centers for Health Statistics. The National Health and Nutrition Examination Survey. Audiometry manual 2003-2004. Available at http://www.cdc.gov/nchs/data/nhanes/nhanes_03_04/AU. pdf Accessed 3/1/2011

Centers for Disease Control and Prevention (2004c). National Centers for Health Statistics. The National Health and Nutrition Examination Survey. Dermatology procedures manual 20032004. Available at http://www.cdc.gov/nchs/data/nhanes/ nhanes_03_04/dermmanual_03_04.pdf. Accessed 3/1/2011

Centers for Disease Control and Prevention (2005) NHANES Analytic and Reporting Guidelines. Available at www.cdc.gov/ nchs/data/nhanes/nhanes_03_04/nhanes_analytic_guidelines_dec_2005.pdf. Accessed 3/1/2011

Conlee JW, Abdul-Baq KJ, McCandless GA, Creel DJ (1986) Differential susceptibility to noise-induced permanent threshold shift between albino and pigmented guinea pigs. Hear Res 23:81-91

Conlee JW, Abdul-Baol KJ, McCandless GA, Creel DJ (1988) Effects of aging on normal hearing loss and noise-induced threshold shift in albino and pigmented guinea pigs. Acta Otolaryngol 106:64-70

Conlee JW, Gill SS, McCandless PT, Creel DJ (1989) Differential susceptibility to gentamicin ototoxicity between albino and pigmented guinea pigs. Hear Res 41:43-51

Conlee JW, Gerity LC, Westenberg IS, Creel DJ (1994) Pigmentdependent differences in the stria vascularis of albino and pigmented guinea pigs and rats. Hear Res 72:108-124

COOPER JC JR (1994) Health and nutrition examination survey of 1971-75: part I. Ear and race effects in hearing. J Am Acad Audiol 5:30-36

Creel D, Garber SR, King RA, Witkop CJ JR (1980) Auditory brainstem anomalies in human albinos. Science 209:1253-1255

FitzPatrick TB (1988) The validity and practicality of sun-reactive skin types I through VI. Arch Dermatol 124:869-871

Garber SR, Turner CW, Creel D, Witkop CJ Jr (1982) Auditory system abnormalities in human albinos. Ear Hear 3:207-210

Gonzalez FJ, Martinez-Escaname M, Munoz RI, Torres-Alvarez B, Moncada B (2010) Diffuse reflectance spectrophotometry for skin phototype determination. Skin Res Technol 16:397-400

Helzner EP, Cauley Ja, Pratt SR, Wisniewski SR, Zmuda JM, Talbott EO, De Rekeneire N, Harris TB, Rubin SM, Simonsick EM,
Tylavsky FA, Newman AB (2005) Race and sex differences in age-related hearing loss: the Health, Aging and Body Composition Study. J Am Geriatr Soc 53:2119-2127

Hong CK, Lee MH, Jeong KH, Cha CI, Yeo SG (2009) Clinical analysis of hearing levels in vitiligo patients. Eur J Dermatol 19:50-56

Hood JD, Poole JP, Freedman L (1976) The influence of eye colour upon temporary threshold shift. Audiology 15:449-464

IshiI EK, TALBotT EO (1998) Race/ethnicity differences in the prevalence of noise-induced hearing loss in a group of metal fabricating workers. J Occup Environ Med 40:661-666

Jerger J, Jerger S, Pepe P, Miller R (1986) Race difference in susceptibility to noise-induced hearing loss. Am J Otol 7:425-429

Lin FR, Niparko JK, Ferrucci L (2011a) Hearing loss prevalence in the United States. Arch Intern Med (in press)

Lin FR, Thorpe R, Gordon-Salant S, Ferrucci L (2011в) Hearing loss prevalence and risk factors among older adults in the United States. J Gerontol A Biol Sci Med Sci 66:582-590

Murillo-Cuesta S, Contreras J, Zurita E, Cediel R, Cantero M, Varela-Nieto I, Montoliu L (2010) Melanin precursors prevent premature age-related and noise-induced hearing loss in albino mice. Pigment Cell Melanoma Res 23:72-83

OHLemiller KK (2009) Mechanisms and genes in human strial presbycusis from animal models. Brain Res 1277:70-83

Ohlemiller KK, Rice ME, Lett JM, Gagnon PM (2009) Absence of strial melanin coincides with age-associated marginal cell loss and endocochlear potential decline. Hear Res 249:1-14

Riley PA (1997) Melanin. Int J Biochem Cell Biol 29:1235-1239

ROBERTS WE (2009) Skin type classification systems old and new. Dermatol Clin 27:529-533, viii

TAYLOR SC (2002) Skin of color: biology, structure, function, and implications for dermatologic disease. J Am Acad Dermatol 46: S41-S62

ToRiello HV (2002) Pigmentary anomalies and hearing loss. Adv Otorhinolaryngol 70:50-55

WolfF D (1931) Melanin in the inner ear. Arch Otolaryngol 14:195

Wolters FL, Klis SF, de Groot JC, Hamers FP, Prieskorn DM, Miller JM, Smoorenburg GF (2003) Systemic co-treatment with alphamelanocyte stimulating hormone delays hearing loss caused by local cisplatin administration in guinea pigs. Hear Res 179:53-61

Wolters FL, Klis SF, Hamers FP, De Groot JC, Smoorenburg GF (2004) Perilymphatic application of alpha-melanocyte stimulating hormone ameliorates hearing loss caused by systemic administration of cisplatin. Hear Res 189:31-40 